\title{
La crisis económica y los servicios bibliotecarios y de información en México
}

\section{RESUMEN}

Rosa Ma. Fernández

La cri sis eco nó mi ca que ha su fri do el país en la úl ti ma dé ca da ha in ci di do en la dis mi nu ción del gas to pú bli co. Esto ha re per cu tido, obviamen te, en las ac ti vi da des edu ca ti vas, cul tu ra les y de in ves ti ga ción y se refle ja en di ver sos apo yos que nece si tan al gu nos servicios, en tre los que se en cuen tran las bi blio te cas. Se men cio nan va rios as pec tos de los serviciosbibliotecarios que han sido afectados duramente por la crisis.

Sin em bar go, a pe sar de los pro ble mas que la cri sis ha cau sa do, en este pe rio do se han te ni dologros biblio te coló gi cos an tes no alcanzados, los cuales son mencionados.

\section{SUMMARY}

The economic crisis in Mexico has imposed a drastic reduction in public spending that has proveked cut backs in education as well. This ac tion has re per cus sions which are to be felt in every as pect and on every le vel of na tio nal edu ca tion, re search and cultu ral ac ti vi ties. Li bra ries as one among the se have been par ti cu lary affected. Some as pects of li brary and in for ma tion ser vi ces serioursly affected are mentioned.

Nevertheless in this period of economic crisis there has been some library achievements never before reached.

Ciertamente la crisis económica no es privativa de México, todos los países la es tán pa de cien do de ma ne ra es pe cial, pero los proble mas que es ta mos en fren tan dolos mexica nos ac tualmente son el efecto de una de las cri sis eco nó mi cas más agut das que ha padecido nuestro país.

Por cri sis, dice un ex per to, se en tien de la si tua ción crea da en México a partir de 1983 por una multitud de factores internos y externos, algunos muy antiguos como nuestra estructura política monopartidista desde hace más de cincuenta años, nues tra eco no mía de cor te ca pi ta lis ta pero sin ca pi tal, la corrupción, la falta de capacidad técnica y profesional para competir en un mundo altamente competitivo y otras más recientes como la devaluación del peso, la inflación, la caída de los precios del petróleo, la nacionalización de la banca, la gran deuda ex ter na, el de sem pleo, la fuga de ca pitales, etcétera.

El crecimiento de las actividades del Estado y una notable am pliación del pre su pues to des ti na do a la edu ca ción su perior mantuvieron la flexibilidad de la estructura social en los seten tas. Este pe río do de auge creó en la cla se me dia ex pec ta tivas que se convirtieron después en desilusión. Hacia 1981 la caída del petróleo fue el primer augurio de que el período de cre ci mien to ha bía lle ga do a su fin, en 1982 se clau su ró el auge con la nacionalización de la banca, la fuerte devaluación del peso, la inseguridad y la desconfianza.
Mé xi co ha bía sos te ni do la pa ri dad de 12.5 pe sos por un dó lar durante 22 años, siendo un ejemplo de estabilidad monetaria en América Latina, pero de 1976, año en que se devaluó por vez pri me ra, pasó a las enor mes de va lua cio nes de los ochentas, hasta llegar a la situación actual en la que el dólar cuesta un peso más cada día y la co ti za ción a fi nes de mar zo de 1989 era de 2,370 pesos por dólar.

Todo esto ocu rrió bajo una ace le ra ción de la in fla ción y un deterioro de la economía nacional, así como un grave crecimien to del de sem pleo. A partir de 1982 y 1983 el cre ci mien to del produc toin ter no bru to (PIB) re gis trócrecimien tos ne gativos de 0.5 y $5.3 \%$, así el pro duc to per cá pi ta de 1984 fue igual al de 1979 , esto es, cinco años de estancamiento. ${ }^{1}$

El millón de mexicanos que cada año in gre san al mercado de trabajo, toca a las puertas de una economía que por primera ocasión en cincuenta años ha decrecido en vez de crecer. El desempleo de la población económicamente activa era del $8 \%$ en 1982 , del $15 \%$ en $1985^{2}$, y del $27.4 \%$ en 1988 .

Los ingresos de los asalariados se han visto constantemente dis minui dos, es así que los sa la rios míni mos han per di do $60 \%$ del po der ad qui si ti vo de 1982 a la fe cha. Al fi nal de esta dé cada los me xi ca nos so mos más po bres que al ini cio del pe río do y se ha acen tua do la de si gual dad en la ya muy de si gual so ciedad en que siem pre he mos vi vi do. De 1976 a 1982, para sa lir delsubdesarrollo y con base en el auge petrolero, México pi- 
dió dinero prestado como nunca antes lo había hecho y se lo prestaron también con una facilidad inconcebible. La deuda pasó de 19 mil mi llo nes de dóla res en 1976 a 80 mil mi llo nes en $1982^{3}$ y a más de 100 mil millones en la actualidad. En poco tiem po la pro me sa de la pros pe ri dad se trans for mó en la cer ti dum bre de una cri sis sin pre ce den tes por el com pro mi so del país de pagar la deuda, 10 a 12 mil millones de dólares anuales. ${ }^{4}$ La decisión del gobierno de seguir su política económica conforme al Fondo Monetario Internacional implica la disminución del gasto público y, por tanto, del sector educativo.

El deterioro del gasto educativo ha sido sustancial y se encuen tra es tan ca do des de 1983. Para te ner pun tos de com paración, mencionaremos que a este sector, en 1940, Cárdenas destinó el 16.4\% del gasto federal, Echeverría, en 1971, el $27.8 \%$, de la Madrid, en 1988 el $6.1 \%$ y en 1989 el $5.6 \%$, o sea, el presupuesto más bajo en los últimos 70 años.

Para ilustrar mejor esta situación es conveniente señalar la proporción del presupuesto para educación con respecto al producto interno bruto, lo que permite comparar con otros países y con el 8 por ciento que recomienda la UNESCO. En 1986, Mé xi co des ti nó el $1.87 \%$ del PIB mien tras que en Cuba fue de $6.3 \% .{ }^{5}$ En 1988 el go bier no fe de ral de di có el $2.6 \%$ ocupando México uno de los últimos lugares de América Latina en este aspecto, el decimoséptimo. ${ }^{6}$

Esto re per cu te ob via men te en to dos los as pec tos y ni ve les de las actividades edu cati vas, cul tu ra les y de in ves ti ga ción einci de en los apo yos que ne ce si tan para lle var se a cabo en tre los que se encuentran las bibliotecas. La Universidad Nacional Au tó no ma de Mé xi co (UNAM), por ejem plo, que es don de se realiza más del $70 \%$ de la investigación científica mexicana, ha per di do du ran te los años ochen ta el $50 \%$ de su pre su pues to real. El Consejo Nacional de Ciencia y Tecnología (CONACyT) tam bién ha te ni do que re cor tar en cer ca del $50 \%$ los apoyos y proyectos de investigación. ${ }^{7}$ En Mé xi co se gas ta en cien cia y tec no lo gía 0.2 y 0.3 por cien to del PIB, mien tras que en los países de sa rrolla dos se des ti na en tre el 2 y 3 por cien to. ${ }^{8}$

Como ya dijimos, la contracción económica que atraviesa el país afec ta to dos los as pec tos de la vida de los me xi ca nos y las medidas restrictivas han repercutido significativamente en las áreas de servicio donde se sitúan las bibliotecas.

Hay que recordar que la crisis tiene dos facetas, una desagrada ble: me nos di ne ro para ha cer cual quier cosa y otra que es el reto de alcanzar soluciones óptimas en menos tiempo y con menos recursos.

La crisis ha afectado duramente varios aspectos de los servicios bibliotecarios y de información, pero dos son los que en especial mencionaremos:

1. Los sa la rios del per so nal que han per di do com pe ti ti vi dady poder adquisitivo, $\mathrm{y}$
2. Los presupuestos para la adquisición de materiales bibliográficos, au dio vi suales, equi po, mo bi lia rio y cons truc ción de edificios.

Los salarios de los biblio te carios, tan to ad mi nis tra ti vos como académicos, han sufrido disminuciones considerables; por ejemplo, en 1986 los salarios de bibliotecarios universitarios habían perdido más del 60 por ciento del poder adquisitivo que te nían en 1978. Ac tual men te son po cos los bi blio te ca rios que ga nan el equi va len te a mil dó la res men sua les, sólo aquellos que ocupan cargos directivos rebasan esta cantidad. El pro me dio ge ne ral va de 300 a 500 dó la res men sua les para bibliotecarios con experiencia, para los re cién egre sa dos de las es cue las es de 150 a 200 dó la res. ${ }^{9}$ Esta si tua ción se agra va en las bibliotecas de los Es ta dos pues los sa la rios que se ofre cen son más bajos que los que se pagan en el Distrito Federal. A todo esto hay que añadir la con ge la ción de pla zas, la no apertu ra de pla zas nue vas y la fal ta de cur sos para el me jo ra mien to profesional a tra vés de cur sos de ca pa ci ta ción y de ac tua li zación, lo que se re fle ja en el de sem pe ño del per so nal al pro porcionar los servicios.

El otro ren glón más afec ta do, que es el pre su pues to de ad quisi ciones, se ha vis to li mi ta do tan to por la res tric ción eco nó mica, como por la de va lua ción. La in fla ción de los pre cios de los li bros y re vis tas es preo cu pan te. En 1986 se es ti mó que el aumen to del pre cio de los li bros cien tífi cos a ni vel in ter nacional de 1980 a 1986 fue del $100 \%$ y que, si bien en el área cien tí fi ca de la UNAM se incrementó el presupuesto 16 veces en seis años, ésto sólo per mi tió ad qui rir el mis mo nú me ro o can ti dad de títulos de libros. "Esta limitación en la compra de mayor número de libros es lo que se considera crisis presupuestal para adquirir material bibliográfico... toda vez que vivimos en la era de la explosión editorial y de la información." ${ }^{10}$

Pero para las bibliotecas universitarias de los Estados, la situación que viven las universidades del Distrito Federal, en especial la UNAM, no es de crisis; así, bibliotecarios de provin cia di cen que "se re fuer za la idea de que es ta mos ha blan do de países diferentes uno desarrollado y uno primitivo... Para las bibliotecas universitarias de provincia la austeridad es algo con lo que se ha te ni do que li diar des de hace mu cho tiempo". ${ }^{11}$ "de igual for ma creo que esas bi blio te cas uni ver sita rias y sus administradores se enfrentaron desde tiempo atrás, no sólo des de hace tres años, con una pe ren ne cri sis pre ci sa mente por la insuficiencia de los recursos económicos, aspecto casi 'normal' de los presupuestos estatales y federal para las universidades públicas" ${ }^{2}$, situación que se ha agravado con esta crisis económica ya que los recursos para adquisiciones en 1986 se redujeron en un $50 \%$ en el mejor de los casos.

Para ejem pli fi car un poco más este as pec to, se ña la re mos que la adquisición de material bibliográfico en las bibliotecas de la UniversidadNacional Au tóno ma Me tro politana, la se gunda uni ver si dad pú bli ca más im por tan te del país ubi ca da en el Dis tri to Fe de ral, refle ja cla ra men te los efec tos de la crisis. De 1974, año en que fue ron fun da dos los plan te les, a 1984 ad quirieron un promedio de 10 mil volúmenes de libros anuales; así, en la Unidad Azcapotzalco, en 1984, se celebró la adqui- 
sición del volumen 100 mil. De 1984 a 1988 se adquirió únicamente un promedio de mil vo lú me nes por año, lo que hace que actualmente sus colecciones cuenten con 115 mil volúmenes aproximadamente. ${ }^{13}$ La suscripción de revistas también de cre ció y la ad qui si ción de ma te rial au dio vi sual es inexistente. Esta situación también la han vivido las bibliotecas especializadas, tanto en lo concerniente al personal como al presupuesto para adquisiciones.

La adquisición de equipo y mobiliario y la construcción de edificios parabiblio te cas se han vis to tam bién du ramen te castigadas. El apo geo de cons truc ción de edi fi cios que se dio en la dé ca da de los se ten tas se vio de te ni do brus ca men te. Sólo en bibliotecas públicas se construyeron algunos edificios y se reacondicionaron edificios antiguos tanto en la capital como en los Estados.

Pero a pe sar de lo que has ta aquí se ha di cho, en este pe río do se han tenido lo gros an tes no al can za dos, como es el caso del esplendor de las bibliotecas públicas.

Quizá el avan ce más sig ni fi ca ti vo de la bi blio te co lo gía me xicana en esta déca da ha sido el Pro gra ma Nacional de Bibliotecas Públicas puesto en marcha por el Presidente de la Repúbli ca el 2 de agos to de 1983, en ple na cri sis. En ese año el país contaba con 351 bibliotecas públicas para atender a una población de casi 77 millones de habitantes, por tan to se re quería de mecanismos que permitieran brindar a los mexicanos mayores oportunidades de acceso gratuito a la lectura, entre otras ra zo nes por el in cre men to del pre cio de los li bros, a través de la prestación de servicios bibliotecarios suficientes y adecuados en toda la República. Delos 2,378 mu ni ci pios del país, sólo 243 tenían bi blio te cas pú bli cas. Por esto el Pro gra ma Nacional de Bibliotecas Públicas se propuso, por medio del es ta ble ci mien to de la Red Na cio nal de Biblio te cas Pú blicas, proporcionarservicios bibliote carios a to dos los mu nicipios del país que con taran al me nos con una es cue la se cun daria.

El progra ma se pla neódes cen tra liza do, esto es, fun da do en la colaboración de los gobiernos federal, estatales y municipales, para lo cual se firmaron convenios de colaboración entre la Se cre ta ría de Edu ca ción Pública, re pre sen tan te del go bierno fe de ral, y los go bier nos de los 31 Es ta dos y el Dis tri to Fede ral. De esta ma ne ra co rres pon dió a la SEP, a tra vés de la Direc ción Ge ne ral de Biblio te cas, co or di nar la Red Na cional de Bibliotecas Públicas, suministrar las colec ciones de libros ya catalogados y clasificados y con sus juegos de tarjetas para formar los catálogos, capacitar al personal necesario para otorgar los servicios y establecer las normas técnicas para el funcionamiento de las bibliotecas. La selección de los títulos de las colecciones, así como la política de servicios, también fueron responsabilidad de la Dirección. Se enfatizó en la estantería abierta y en el préstamo a domicilio.

Para diciembre de 1988 el nú me ro de bi blio te cas se ha bía incre men ta do de 351 a 3,047, esto es, 2,696 bi blio te cas abier tas en cinco años. En este período el incremento de volúmenes que integran el acervo bibliográfico de las bibliotecas públi- cas fue con si de ra ble: en 1982 ha bía 1'332,000 vo lú me nes, en 1988 al término del Programa se contaba ya con 11'042,862 volúmenes. ${ }^{14}$

En 1987 se emitió un decreto por el que se consolidó la Red Na cio nal de Biblio te cas Públicas. Es asíque, gra cias a la co labo ra ción de los tres ni ve les de go bier no y a que se tuvo el apoyo po líti co, pre su pues tal y le gal, se cum plió con el Pro gra ma Nacional de Bibliotecas Públicas. La Dirección General de Bibliotecas, que ahora depende del ConsejoNacional para la Cul tu ra y las Ar tes se debe abo car a vi gi lar el buen fun cio namien to de la Red y a eva luar las co lec cio nes y al per so nal que son los puntos débiles de las bibliotecas del Programa.

La Dirección General de Bibliotecas ha tenido otro aporte significa tivoalde sarrollobiblio te cario de Méxi cocon su programa de publicaciones; éste comprende varias series como las de manuales, historia general de las bibliotecas en México, his to ria de las bibliote cas es ta tales, te mas de biblio te cología, di rec to rios, le yes y re gla men tos. En to tal, a fi nes de 1989 había publicado 45 títulos.

Un avance en los servicios de información en esta década lo constituye la creación de bancos nacionales de información aus pi cia dos por el CONACyT y dis po ni bles para to das las bibliotecas del país conectadas al Servicio de Consulta a Bancos de Información (SECOBI).

En México se inició la consulta a bancos de información en 1975 cuan do se es table ció SECOBI en elCONACyT, a tra vés del cual las bibliotecas afiliadas consultaban los servicios de in formación au to ma tiza dos dis ponibles en el mun do. Sinembargo, la información bibliográfica y documental generada en el país en las di fe ren tes áreas del co no ci mien to no era aprovechada por carecer de las obras de consulta (bibliografías e índices) impresos o automatizadosnecesarios para recuperar la información nacional publicada en revistas, congresos, o bien, las patentes, nor mas, es ta dís ticas, etc. Exis tían al gu nos ín di ces im pre sos pero con atra so en su pu bli ca ción y ca re cían de volúmenes acumulativos.

En 1984, CO NACyT fijó en uno de sus pro gra mas re la cio nadoscon lain for mación cien tíficay tec noló gicala for mación y el apoyo para la "creación de bancos de datos nacionales orientados a las necesidades del país, fomentando su utilización eficiente entre los usuarios potenciales". Cabe aclarar que se en tien de por ban cos de in for ma ción tan to a las ba ses de datos que contienen información bibliográfica como a los ban cos de da tos que con tienen in for ma ción de tiponu mérica.

Actualmente se encuentran en operación y a disposición del pú bli co usua rio de SE CO BI, 31 ban cos y hay otros 20 en proce so de de sa rrollo. Des ta can en tre los prime ros SIE-BANXICO, Sistema de Información Económica-Banco de México; UNAM-JURE, Sistema de Información sobre Legislación Nacional elaborado por la UNAM; el Catálogo Colectivo de Publicaciones Seriadas, compilado por el CONACyT; PERIODICA, Indice de Re vistasLatinoamericanasenCiencias; SECOFI-NOM, Sistema de Información de Normas Oficia- 
les Mexicanas; BIDE-RSRE, Banco de Información sobre Desarrollo Social y Regional; BIVE, Banco de Información de Medicina Veterinaria y Zootecnia; CLASE, MEXICOAR TE, Ban cos sobre Patentes, Cen sos, Investi ga ción Cientí fica y Tecnológica, etcétera.

La mayoría de estos bancos han sido generados por organismos gubernamentales. En México, como en otros países, el sec tor pú bli co y gu ber na men tal es el que le ha dado un ma yor impulso e importancia a los bancos de información. En relación con los te mas de los ban cos, los que más abun dan son de ciencia y tecnología, siguen los de economía, después los de humanidades y finalmente, los interdisciplinarios.

Otro logro en los servicios bibliotecarios mexicanos ha sido laau tomatización de sistemasbibliotecariosuniversitarios.la crisis económica ha obligado a los bibliotecarios a ser más produc ti vos y crea ti vos para de sa rrollar, adap tar y utilizarlos adelantos de la tecnología. Esta sigue evolucionando en los campos de al macena mien to y recu peración de lain for mación y los cam bios que se pre sen tan son un reto para los bi blio te carios. En México, además del uso de los grandes equipos de computación, las microcomputadoras están modificando el medio ambiente de las bibliotecas y el CD-ROM empieza a jugar un papel importante en la transferencia de la información.

La primera universidad que introdujo la computación en sus servicios bibliote cariosfue la Universidad Nacional Au tónoma de Méxi co al po ner en ser vi cio el sis te ma LIBRUNAM en 1978 con el fin de registrar en forma electrónica los acervos existentes en las distintas bibliotecas universitarias y la optimi za ción de los pro ce sos téc ni cos de las obras mo no gráficas, clasificación, producción de tarjetascatalográficas y actualización de inventarios de todos los libros adquiridos por las 164 bibliotecas y colecciones en la UNAM.

En la actualidad, el sistema cuenta con cerca de 400 mil títulos de mo no grafías, los cua les su pe ran los dos millo nes de volúmenes. La Direc ción Ge ne ral de Biblio te cas es la res pon sable del desarrollo de LIBRUNAM. El sistema sigue el for ma to MARC II y los me dios para re cu pe rar la in for ma ción en lí nea son a tra vés de cual quie ra de los ele men tos de la fi cha catalográfica, así como por cualquier palabra que se encuentre en el contenido finalde laficha. La re cu pe ra ción pre sen ta una mo da li dad es pe cial, es fo né ti ca, o sea que se pue de re cur perar lain for ma ción con errores me ca no gráfi cos y or to gráficos en las pa la bras que se uti li zan para la re cu pe ra ción de datos. Las bibliotecasdepartamentales tienen acceso al sistema a través de una terminal, con lo cual se ha establecido ya una red automatizada de bibliotecas de la UNAM. LIBRUNAM se maneja con una máquina Britton-Lee IDM, acoplada a un computador Alpha-micro.

Tan to el ac ce so en línea como la edi ción de mi cro fi chas tu vieron una cre cien te de man da de uso, por lo que lle va ron a la Dirección General de Bibliotecas a explorar nuevas tendencias y tecnologías que permitieran cubrir estas necesidades. Con la introducción de las microcomputadoras en las bibliotecas mexicanas y el desarrollo de los sistemas ópticos de almacenamiento y recuperación de la información, se encontraron posibilidades interesantes para solucionar este problema. La DGB es tu diólas ven tajas, cos tos y caracterís ticas del al macena mien to por me dio delCD-ROM (Com pact Disc-Read Only Memory) o disco compacto y decidió que era adecuado para lasne cesida des del sis te maLIBRUNAM, con for man do asíel primer banco de datos bibliográficos producido en México y América Latina con esta tecnología. Las ventajas del uso de LIBRUNAM a tra vés del dis co com pac to son múl ti ples, en tre otras: tener el registro del acervo de las bibliotecas de la UNAM cuan do se de see, ra pi dez en las bús que das, po der copiar la información que se requiere. La infraestructura del equipo necesario es mínima.

Otros módu los que con for man el sis te maLIBRUNAMyaestán en fun cio na mien to. Es tos son el mó du lo au to ma ti za do de circulación que ope ra en la Bi blio te ca Cen tral que fun cio na a base de lectores ópticos y código de barras y del cual existe una ver sión para mi cro com pu ta do ras PC para su uso en las bibliotecas departamentales. Igualmente, ya funciona el banco de da tos de te sis, TE SIU NAM que se ocu pa de re gis trar to das las tesis de licenciatura, maestría y doctorado de la UNAM a partir de 1914, así como de 29 universidades incorporadas y 17 independientes como El Colegio de México, la Universidad Autónoma Metropolitana y el ITAM.

Ac tual men te el ban co tie ne cer ca de 100 mil re gis tros de tí tur los correspondientes a las tesis de 1979 a la fecha. La información se puede recuperar por cualquier palabra del título, del autor, por carrera, escuela y asesor de tesis. Se encuentra disponible a través de SECOBI.

Otro mó du lo en fun cio nes es SERIU NAM que com pren de 16 mil re gis tros de pu bli ca cio nes pe rió di cas exis ten tes en las bibliotecas de la UNAM.

La Universidad de Colima también ha automatizado sus servicios bibliotecarios, para lo que ha desarrollado un sistema llamado SIABUC integrado por cinco módulos: control de ad qui siciones, controlde análi sis bibliográfico, con trolde archi vos de con sul ta, con trol de acer vos y ser vi cio de prés ta mo e información estadística. El sistema fue desarrollado por la Dirección General de Desarrollo Bibliotecario y ha utilizado un equipo micro de los identificados como compatibles que si gue el for ma to MARC. Des de fi nes de 1989 está dis po ni ble la ver sión en CD-ROM que con tie ne un ca tálo go co lec ti vo de 82 mil registros. Este disco compacto comprende también el Catálogo Colectivo de Publicaciones Seriadas de CONACyT, bibliografía del Estado de Colima, etc.

Actualmente, cercade 70 bibliotecasuniversitarias yespecializadas están utilizando SIABUC. El software es gratuito. SIABUC cuenta con el apoyo de la Subsecretaría de Educación Superior de la SEP.

Otroavancesignificati vo de labibliote cología mexicanaes la creación del Centro Universitario de Investigaciones Bibliotecológicas (CUIB) en la UNAM, fundado en 1981 con la fi- 
nalidad de realizar investigación que ayude a los problemas nacionales re laciona dos con las bi blio te cas y cen tros de in formación y pro por cio nar apo yo a la edu ca ción, la in ves ti ga ción y la di fu sión de la cul tu ra. Este cen tro, úni co en Améri ca La tina, en la actualidad cuenta con 18 investigadores de tiempo completo y ha publi ca do ya 27 títu los de obras de bi blio te colo gía pro duc to de las in ves ti ga cio nes rea li za das y tam bién ha he chocoediciones con otras ins titu ciones es pecializa das. $\mathrm{Pu}$ bli ca la úni ca re vis ta que ac tual men te se edi ta en Mé xi co sobre bibliotecología: Investigación Bibliotecológica.

Como podemos ver, esta década de crisis ha sido un período de progresosignifica ti vo para la profe sión y tes ti go de importantes desarrollos de la bibliotecología mexicana.

\section{NOTAS}

1. México Mañana. Héctor Aguilar Camín et al. México, Oceano- Nexos, 1986. p. 41.

2. Ibid. p. 42 .

3. Ibid. p. 197.

4. Ibid. p. 198.

5. Francisco Már quez Páez. Pre sen ta ción, en El Impacto de la Crisis en la Biblioteca de enseñanza superior. Monterrey, ABIESI, 1987. p. 23.

6. ManuelPeimbert. Investigacióne IdentidadNacionalen Conferencias Temáticas. Tema5. Mé xico, UNAM, 1990. p.32.

(Cuadernos del Congreso Universitario, 16)

7. México Mañana. Op. cit. p. 224

8. Manuel Peimbert. Loc. cit.

9. Da tos ob te ni dos por en tre vis tas per so nales a alum nos y en con voca to rias de la UNAMy otras instituciones.

10. Elsa Barberena. Las Co lec ciones Docu men ta les del Sub sis te ma de la In ves ti gación Científicaen la UNAM ante la Crisis Económica, en El Impacto... p.131.

11. J. Alberto Arellano. Comentario, en El impacto... pp. 149, 151.

12. He len La drón de Gue va ra. Mesa Re don da so bre la Ad mi nis tra ción de Bi blio te cas Uni versitarias en Epoca de Crisis, en Memorias. XVII. Jornadas Mexicanas de Biblioteconomía. Puebla 1986. México, AMBAC, 1988. p. 435.

13. Entrevista con el Jefe de Adquisiciones de la COSEI de la UAM-A.

14. ElProgramaNacionalde Bibliotecas Públicas 1983-1988...-Méxi co, SEP.Dirección Ge ne ral de Bibliotecas, 1988 . p. 39.

\section{OBRAS CONSULTADAS}

Aranda R., Josué. Los Recursos Humanos Bibliotecarios ante la Crisis Económica y Estrategia Na cio nal para la Edu ca ción Su pe rior, en El Im pac to de la Cri sis en las Bi blio te cas de En señanza Superior. México, ABIESI, 1987. p.69-80.

Arellano, J. Alberto. Comentario, en El Impacto... Op cit p. 149-154.

Banco de Datos Bibliográficos LIBRUNAM/CD-ROM. UNAM, Dirección General de Bibliotecas, 1988. 10p.

Barberena Blásquez, Elsa. Las Colecciones Documentales del Subsistema de la Investigación Científica en la UNAM ante la Crisis Económica, en El Impacto... Op. cit. p. 129-147. 
La drón de Gue vara, Hellen. Mesa Re don da so bre la Ad mi nis tra ción de Biblio te cas Uni versitarias en Epoca de Crisis, en Memorias. XVII Jornadas Mexicanas de Biblioteconomía. Puebla, 1986. México, AMBAC, 1988. p. 431-442.

México Mañana. Héctor Aguilar Camín et al. México, Oceano- Nexos, 1986. 231p.

Panorama General de los Servicios Bibliotecarios y de Información en México. CUIB/UNAMINFOTEC. Primer Seminario sobre Políticas Nacionales de Información para la Investigación y el Desarrollo. México. CONACyT, 1987. 63 p. (Documento de trabajo, 2)

Peimbert, Ma nuel. In ves ti ga ción e Iden ti dad Na cional, en Conferencias Temáticas. Tema5. México, UNAM, 1990. p. 32- 36.

El Pro grama Na cional de Biblio te cas Públicas 1983- 1988 yel Cen tro Biblio te ca rio Nacio nal de BibliotecasPúblicasdeMéxico. México, SEP.Direc ción Ge neralde Bibliote cas, 1988.67 p.

Rodríguez, Victorico y Lourdes Feria. Automatización de las Funciones Bibliotecarias a través de SIABUC. Colima. Universidad de Colima, 198? 12h.

VelázquezMerlo, Fernando. Estructura, Funcióny Contenidodelos Bancos Nacionales de Información. México, 1987. 240 p. Tesis ENBA

Vouts sas M., Juan. El Sis te ma TE SIU NAM, en Memorias. XIX Jor na das Me xi ca nas de Bi blio teconomía. Villahermosa, Tab. 1988. México, AMBAC, 1988. p. 113-115.

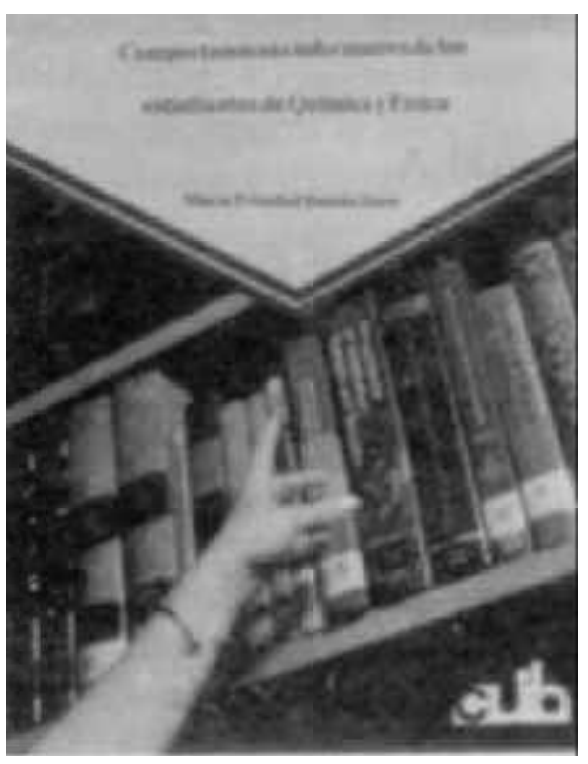

Comportamiento informativo de los estudiantes deQuímicayFísica. Ro mán, Ma. Trinidad116 p.

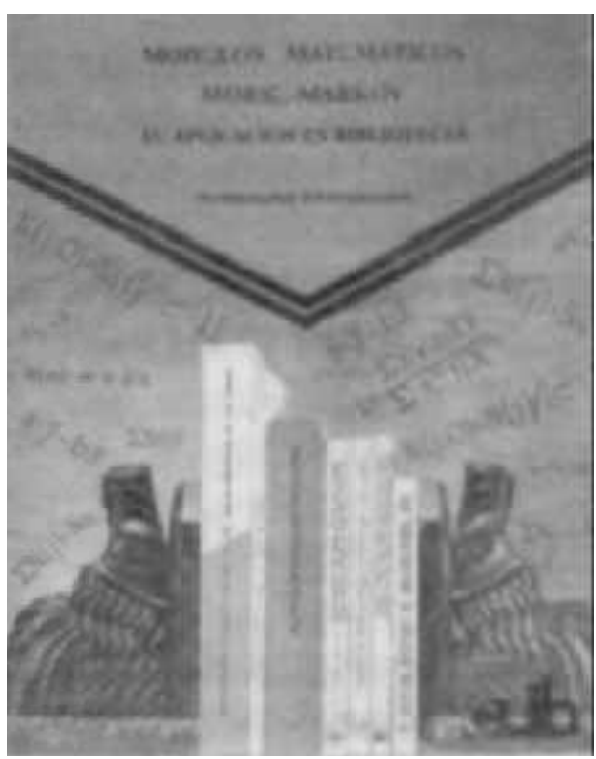

Modelos Matemáticos Morse-Markov. Korranzadeh, Hesh matallah 122 p. 